\title{
Neutron Stars, Strange Pulsars and Strange Dwarfs
}

\author{
Fridolin Weber \\ Institute for Theoretical Physics, University of Munich, Germany \\ Norman K. Glendenning \\ Lawrence Berkeley National Laboratory, Berkeley, CA, U.S.A.
}

\section{Introduction}

The hypothesis that strange quark matter may be the absolute ground state of the strong interaction (not ${ }^{56} \mathrm{Fe}$ ) has been raised independently by Boder and Witten. If the hypothesis is true, then a separate class of compact stars could exist, which are called strange matter stars. The properties of the complete sequence of such stars, which range from compact neutron-star-like strange stars to strange dwarfs to strange planets. The latter two constitute the strange counterparts of ordinary white dwarfs and planets, respectively. The properties of these objects are discussed in this paper.

\section{Properties of strange matter stars}

The main features of strange matter stars can be summarized as follows:

- The complete sequence of compact strange stars can sustain extremely rapid rotation and not just those close to the mass peak, as is the case for neutron stars (Glendenning 1992, Glendenning \& Weber 1992, Weber \& Glendenning 1993).

- From Glendenning \& Weber (1992) it follows that the crustal moment of inertia relative to the star's total moment of inertia, $I_{\text {crust }} / I_{\text {total }}$, varies between $10^{-3}$ and $\sim 10^{-5}$. If the angular momentum of the pulsar is conserved in the quake, then the relative frequency change and moment of inertia change are equal and one arrives at (Glendenning \& Weber 1992)

$$
\frac{\Delta \Omega}{\Omega}=\frac{\Delta I}{I_{0}}>\frac{\Delta I}{I}=\frac{\Delta I}{I_{\text {crust }}} \frac{I_{\text {crust }}}{I} \equiv f \times\left(10^{-5}-10^{-3}\right),
$$

where $I_{0}\left(<I_{\text {total }}\right)$ denotes the moment of inertia of that part of the star whose frequency is changed in the quake, and $f$ is the fractional change of the crust's moment of inertia necessary to account for the glitch. Since observed glitches have $\frac{\Delta \Omega}{\Omega} \simeq 10^{-9}-10^{-6} \Rightarrow f \lesssim 10^{-1}$. Secondly, using the above relation one obtains for the change of the spin-down rate

$$
\frac{\Delta \dot{\Omega}}{\dot{\Omega}}=\frac{\Delta \dot{\Omega} / \dot{\Omega}}{\Delta \Omega / \Omega}(\Delta \Omega / \Omega)=\frac{\Delta \dot{\Omega} / \dot{\Omega}}{\Delta \Omega / \Omega} f \frac{I_{\text {crust }}}{I_{0}}>\left(10^{-1}-10\right) \times f .
$$


Since $\frac{\Delta \dot{\Omega}}{\dot{\Omega}}=10^{-3}$ to $10^{-2}$ from observation (Crab and Vela) $\Rightarrow f<$ $10^{-4}-10^{-1}$ which is consistent with $f \lesssim 10^{-1}$ from above. So the crustal moment of inertia can account for both the relative frequency change and the change in the spin-down rate!

- Strange stars can possess nuclear crusts of thickness $\sim 1 \mathrm{~km}$ to $\sim 10^{3}$ $\mathrm{km}$, depending on central star density (Glendenning, Kettner, \& Weber 1995a,b). This might be of great importance for their cooling behavior (Weber, Kettner, Weigel, \& Glendenning 1995).

- Strange stars possess masses in the range $\sim 2 M_{\odot}$ to $10^{-4} M_{\odot}$ and radii from several kilometers to $\sim 10^{3} \mathrm{~km}$ (Glendenning, Kettner, \& Weber $1995 \mathrm{a}, \mathrm{b})$. Because masses and radii of $\sim 10^{-4} M_{\odot}$ and $\sim 10^{3} \mathrm{~km}$ are completely excluded for both neutron stars as well as white dwarfs, such star properties may serve as additional signatures for hypothetical strange stars!

- If the light, planetary-like strange stars exist and if they are abundant enough in our Galaxy, then the gravitational microlensing experiments should see them!

- We find white-dwarf-like strange stars that owe their stability solely to the strange cores at their centers (strange dwarfs) (Glendenning, Kettner, \& Weber $1995 \mathrm{a}, \mathrm{b})$. They carry nuclear crusts whose density at the base is up to about 400 times higher than the central density in the most massive white dwarf. Hence such strange dwarfs constitute a possible new class of dense stars, if the strange matter hypothesis is correct!

\section{References}

Glendenning, N. K. 1992, PRD, 46, 4161

Glendenning, N. K., \& Weber, F. 1992, ApJ, 400, 647

Weber, F., \& Glendenning, N. K. 1993, Proceedings of the First Symposium on Nuclear Physics in the Universe, ed. M. W. Guidry and M. R. Strayer (IOP Publishing Ltd, Bristol, UK), 127

Glendenning, N. K., Kettner, Ch., \& Weber, F. 1995a, Phys. Rev. Lett., 74, 3519

Glendenning, N. K., Kettner, Ch., \& Weber, F. 1995b, ApJ, 450, 253

Weber, F., Kettner, Ch., Weigel, M. K., \& Glendenning, N. K. 1995, Proceedings of the International Symposium on Strangeness and Quark Matter, Crete, Greece, eds. G. Vassiliadis, A. D. Panagiotou, S. Kumar, and J. Madsen (World Scientific), 308 